\title{
Cold Flow Testing for Liquid Propellant Rocket Injector Scaling and Throttling
}

\author{
R. Jeremy Kenny ${ }^{1}$, Marlow D. Moser ${ }^{2}$, \\ University of Alabama, Huntsville, AL, 35812 \\ James Hulka ${ }^{3}$ \\ Jacobs Sverdrup, MSFC Group, Huntsville, AL, 35812 \\ Gregg Jones ${ }^{4}$ \\ NASA Marshall Space Flight Center, Huntsville, AL, 35812
}

\begin{abstract}
Scaling and throttling of combustion devices are important capabilities to demonstrate in development of liquid rocket engines for NASA's Space Exploration Mission. Scaling provides the ability to design new injectors and injection elements with predictable performance on the basis of test experience with existing injectors and elements, and could be a key aspect of future development programs. Throttling is the reduction of thrust with fixed designs and is a critical requirement in lunar and other planetary landing missions. A task in the Constellation University Institutes Program (CUIP) has been designed to evaluate spray characteristics when liquid propellant rocket engine injectors are scaled and throttled. The specific objectives of the present study are to characterize injection and primary atomization using cold flow simulations of the reacting sprays. These simulations can provide relevant information because the injection and primary atomization are believed to be the spray processes least affected by the propellant reaction. Cold flow studies also provide acceptable test conditions for a university environment. Three geometric scales $-1 / 4$ scale, $1 / 2$-scale, and full-scale - of two different injector element types - swirl coaxial and shear coaxial - will be designed, fabricated, and tested. A literature review is currently being conducted to revisit and compile the previous scaling documentation. Because it is simple to perform, throttling will also be examined in the present work by measuring primary atomization characteristics as the mass flow rate and pressure drop of the six injector element concepts are reduced, with corresponding changes in chamber backpressure. Simulants will include water and gaseous nitrogen, and an optically accessible chamber will be used for visual and laser-based diagnostics. The chamber will include curtain flow capability to repress recirculation, and additional gas injection to provide independent control of the backpressure. This paper provides a short review of the appropriate literature, as well as descriptions of plans for experimental hardware, test chamber instrumentation, diagnostics, and testing.
\end{abstract}

Nomenclature

$\begin{array}{ll}A & =\text { area } \\ d & =\text { diameter } \\ F & =\text { force } \\ L & =\text { length } \\ n & =\text { length scale factor } \\ \operatorname{Re} & =\text { Reynolds number } \\ \mathrm{V} & =\text { Velocity }\end{array}$

\footnotetext{
${ }^{1}$ Graduate Research Assistant; Mechanical and Aerospace Engineering Dept., Technology Hall/N244, Student Member, AIAA.

${ }^{2}$ Associate Research Professor, Mechanical and Aerospace Engineering Dept., Technology Hall/S232, Senior Member, AIAA.

${ }^{3}$ Engineering Specialist, MSFC Group, M/S ER32, NASA MSFC, AL, 35812; Senior Member, AIAA.

${ }^{4}$ Combustion Specialist, M/S ER32, NASA MSFC, AL, 35812.
} 


$\begin{array}{ll}\text { We } & =\text { Weber number } \\ \rho & =\text { density } \\ \mu & =\text { viscosity } \\ \sigma & =\text { surface tension }\end{array}$

\section{Subscripts}

$\begin{array}{ll}e & =\text { exit } \\ f & =\text { fuel } \\ g & =\text { gas } \\ H & =\text { full scale } \\ l & =\text { liquid } \\ M & =\text { sub. scale } \\ o x & =\text { oxidizer }\end{array}$

\section{Introduction}

$\mathrm{W}$ idely advocated as a promising design and development tool in the 1950s, scaling of liquid rocket engines (LREs) has been only used by research programs since then with but a few exceptions. ${ }^{1.2}$ Scaling has been defined as the ability to design new combustors, including injectors and injection elements, with predictable performance on the basis of test experience with existing injectors and injection elements. ${ }^{1,3}$ When used judiciously during LRE development programs, scaled hardware can lower development costs and increase operational margins because much of the development fabrication and testing can be conducted with smaller and less expensive hardware. Testing can be conducted in a lab-scale environment where design parameters are easier to change and sensitivities examined over a wider range. Full-scale design features can then be optimized at lower cost compared to full-scale iteration and design. Recently, these benefits have become even more attractive with growing demands for economical, yet comprehensive, liquid rocket component research.

While the notion of "scaling" can be clearly defined, the definition of "subscale" has proven to be more ambiguous. ${ }^{1,2}$ Generally, a 'subscale' test article has features (geometrical and/or operational) that are reduced in absolute terms from full-scale design features. However, the design of the subscale hardware is often defined by programmatic constraints, so that many of the scaling rules such as described in [1] are not followed. For example, sub-scale sized combustion chambers are often designed with full-scale injector elements; this results in chamber geometrical characteristics not matching the combustion profiles provided by the elements. This lack of consistency is not only the fault of programmatic constraints, however; there is also a general and wide-spread disagreement on how the various LRE processes scale directly with the hardware geometry. This disagreement is especially true for the physics in such processes as propellant injection, atomization, vaporization, mixing, and reaction that define the combustor performance, combustion stability, and heat transfer between the reacting flow and the chamber walls. To date, there have been numerous LRE development programs that have used subscale hardware to some extent (and some of which had some semblance of scaling), but there has been no focused research on how any of these processes scale exactly with engine geometry. An example of the varying use of subscale hardware to predict fullscale design features and behavior in typical LRE combustor development programs is shown in Fig. 1. As another example, dynamic scaling in wind tunnel testing focuses on matching the operational parameters of the full-scale design and not necessarily the geometrical features. ${ }^{4}$ Consequently, the subscale test model may not be visually identical to the full-scale design and physical phenomena linked to full-scale geometry may not be represented during subscale testing.

Geometric scaling, on the other hand, is focused on capturing all of the full-scale geometrical features within the subscale model. Geometrically subscale hardware is based on a universal linear reduction of all full-scale length scales. ${ }^{5}$ For rocket engines, these length scales include chamber lengths and diameters, injector faceplate diameters, and nozzle contour diameters. Non-length parameters, such as the number of injection elements, are maintained throughout the geometric scaling process as well. Thus, a subscale test article will look visually identical to the full-scale hardware, just reduced in physical size. While complete geometric and dynamic similarity 
cannot be preserved for complicated thermo-fluidic systems, ${ }^{1}$ geometric subscale testing can still be used to study a large number of processes inherent within liquid rocket engine combustion.

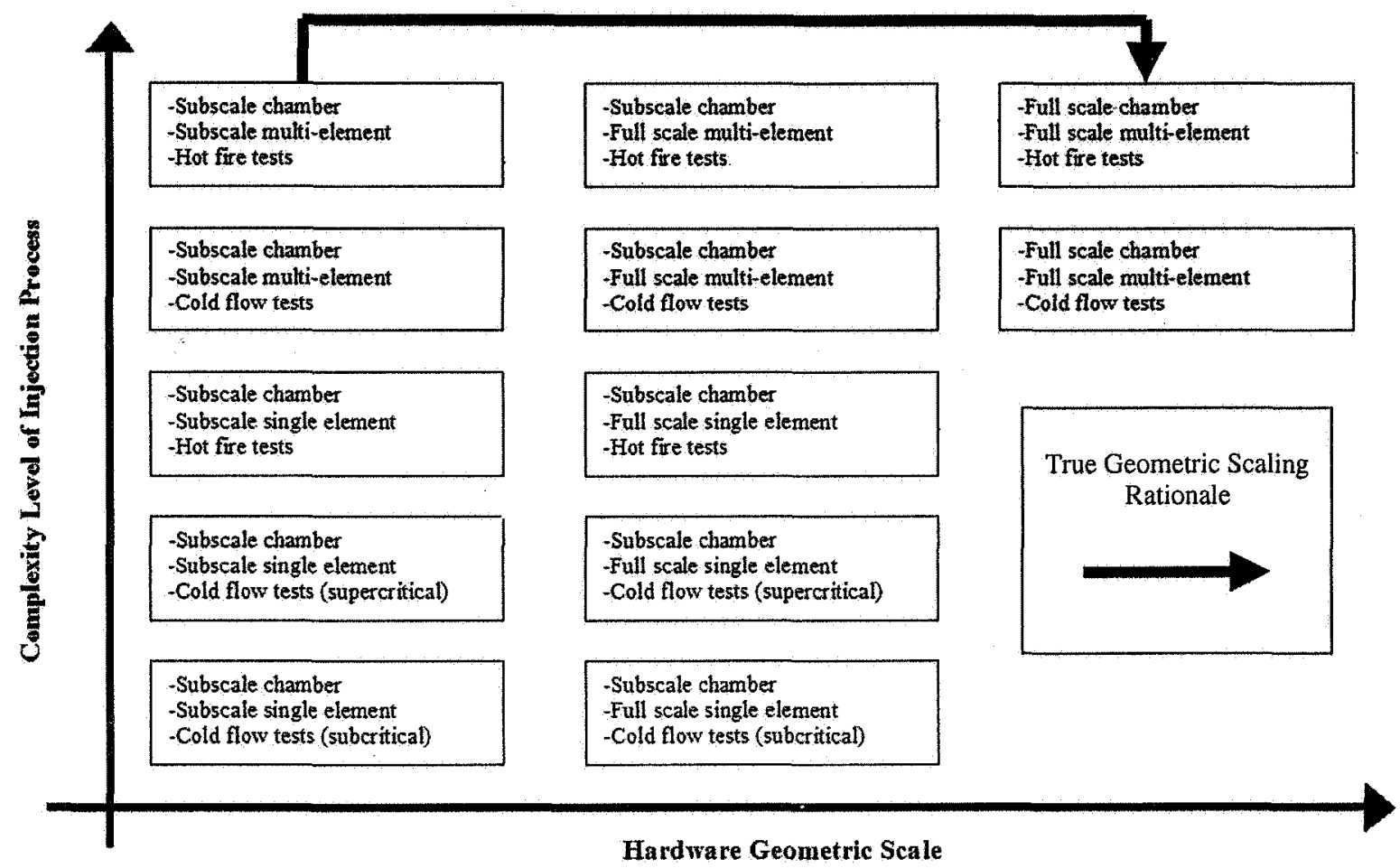

Figure 1. Past LRE injector programs utilizing subscale testing techniques.

Throttling is different from scaling in that flow rates and thrust are reduced while the combustion chamber dimensions and - generally - the injector dimensions do not change. With a fixed combustion chamber throat area, the chamber pressure is reduced proportionally to thrust. Throttling combustors have great importance in vehicles that must maneuver and land, so NASA is interested in this capability for lunar and planetary missions.

\section{A. Geometric Scaling Rationale for Liquid Rocket Engines}

The basic idea of geometric scaling is to reduce all lengths of the full-scale design by a universal linear scale factor $n$, and use the result to generate a scaled model. ${ }^{5}$ The general analytical description is given as Eq. (1), with ' $H$ ' standing for the reference ("full-scale") length, and ' $M$ ' standing for the subscale ("Model") length.

$$
L_{M} / L_{H}=n
$$

Eq. (1) is not limited to physical scale reduction; there are examples of tests run with larger model test articles compared to the full-scale. ${ }^{7}$ However, the typical geometric scaling application is generation of a model smaller than the reference specimen $(n=1 / 2,1 / 4$, etc...). Penner outlined the geometric scaling scheme specific to bipropellant rocket engines. ${ }^{6,8}$ This section will briefly cover the rationale for taking a full-scale rocket design $(H)$, and sizing a subscale test article $(M)$. To start, assume the liquid rocket design to be studied as operating at known chamber pressures, fixed propellant injection temperatures, and optimum expansion. The thrust equation for this system is given as: ${ }^{9}$

$$
F=\rho_{e}^{*} A_{e}^{*} V_{e}^{2}
$$

The ratio of the model thrust and the full-scale or reference thrust is: 


$$
\frac{F_{M}}{F_{H}}=\frac{\left.\rho_{e}^{*} A_{e} * V_{e}^{2}\right|_{M}}{\left.\rho_{e} * A_{e} * V_{e}^{2}\right|_{H}}=\frac{\left.d_{e}^{2}\right|_{M}}{\left.d_{e}^{2}\right|_{H}}=\left(\frac{\left.d_{e}\right|_{M}}{\left.d_{e}\right|_{H}}\right)^{2}=n^{2}
$$

Implicit in Eq. (3) is the assumption that the exit momentum fluxes, $\rho \mathrm{V}^{2}$, between the model and fullscale specimens are the same. This is generally true for fixed chamber pressure and assuming that the overall combustion physics are preserved upon scaling. The exit cross-sectional area is assumed circular, so Eq. (3) shows the only varying quantity sensitive to scale is the exit diameter. Because diameter is a length scale, the model-tofull-scale diameter ratio is simply $\mathrm{n}$, the scale factor. This gives a thrust ratio that changes with the scale factor squared. Like the exit diameters, other rocket chamber length parameters will scale linearly with the same scale factor. However, non-length parameters, such as the number of injection elements, should be preserved upon scaling. For example, the scaling ratios of the number of oxidizer ('ox') and fuel ('f') ports are:

$$
\frac{\left.N_{o x}\right|_{M}}{\left.N_{o x}\right|_{H}}=\frac{\left.N_{f}\right|_{M}}{\left.N_{f}\right|_{H}}=1
$$

While past geometrically scaled hardware may preserve the overall chamber dimensions, the number and size of injector elements have not been strictly geometrically scaled.

This technique of geometric scaling is not sufficient to fully categorize the scaled behavior of the thermofluidic processes: it simply defines dimensions for the model. Spaulding, along with many other authors, ${ }^{1,10-12}$ has yielded nine important dimensionless groups that capture all of the non-reacting and reacting flow physics. Spaulding also states that it is impossible to preserve geometric similarity and all of the dimensionless groupings listed above for the most complex thermo-fluidic system. Because not all dimensional groupings can be preserved upon scaling, research objectives are used to make judicious choices on which physical processes to neglect and which to keep. This allows for certain dimensionless numbers to be ignored during the study and ensures total system similarity within the research goals dictated. So called 'partial modeling' by Spaulding, ${ }^{10}$ this technique supports focused study on particular aspects of complex reacting flows. Details on how partial modeling affects this particular work will be outlined in the next sections.

\section{B. Examination of LRE Combustor Physics for Scaling}

Selecting particular physics for partial modeling within the realm of LRE combustion is challenging because there are dozens of processes and many of them are strongly coupled. The literature provides examples where many of these individual processes are listed and some of their interactions displayed (e.g., [12], [13]). Another example is shown in Fig. $2 .{ }^{15}$ Generally, the major individual processes of a typical LRE combustion flowfield include injection, atomization, vaporization, mixing, reaction, and expansion (or acceleration), occurring typically in that order. However, not all LRE combustion devices have all these processes. Combustion devices with gaseous propellants do not have atomization and vaporization processes, while others, operating at supercritical chamber pressures, have forms of atomization and vaporization that are largely different than what occur at subcritical pressures. ${ }^{16,17}$ Coupling between the processes is shown in Fig. 2 as a physical means in which the processes interact, e.g., reaction affects atomization by expanding gas and increasing turbulence in the local regions where atomization occurs, changing the aerodynamic forces on the liquid structures while they are breaking up.

A significant, research-oriented evaluation of scaling of LRE combustion devices will require evaluation of all the individual processes as well as their interactions. Some of these processes will be easier to investigate than others. The research plan presented in this paper is focused on evaluating the scaling of injection and atomization processes, which appear most tractable to experimental methods readily available to university-level laboratories.

Injection and atomization processes occur first in the preparation of fluids to be reacted and expelled from the combustion chamber for the production of thrust. Among all the processes in the combustion chamber, these would seem to be the least affected by the reaction. Nonetheless, there is always some effect of the reaction, as 
suggested in Fig. 2, and this coupling often depends on the injector element design and propellant combination. Storable propellants with impinging-jet ìnjectors, e.g., are subject to a reactive "blowapart" phenomenon that causes the unlike propellant streams to explosively separate, hence preventing any additional atomization that would have occurred between the interaction of the unlike propellant streams without the reaction. ${ }^{18}$ Coaxial injectors with liquid oxygen and hydrogen or hydrocarbon fuels, on the other hand, do not tend to react so explosively, because the oxygen and/or the hydrocarbon fuel need time to vaporize and mix. These times, the vaporization and mixing processes shown qualitatively on Fig. 2, separate the injection and atomization processes from the reaction, but do not necessarily de-couple them.

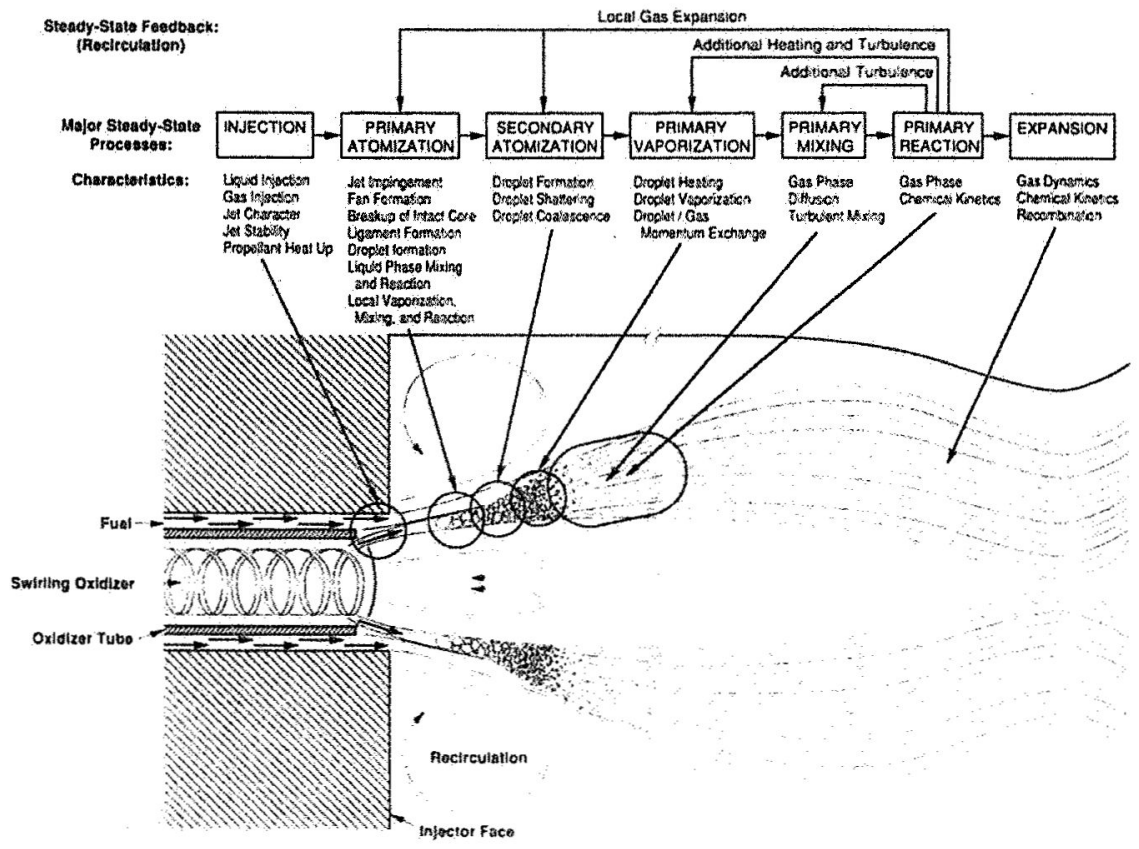

Figure 2. Illustration of relationship of physical processes in the combustion chamber of an LRE.

Atomization itself is a process that occurs over multiple steps. Researchers generally agree that there are at least two: 1) primary atomization, which is the initial disintegration of the jet into ligaments and large droplets, and 2) secondary atomization, which is the breakup of the ligaments and large droplets into small droplets.

Shear coaxial injectors are believed to depend upon the velocity difference between the two fluid streams to provide the primary atomization of the liquid propellant. Swirl coaxial injectors, on the other hand, are believed to depend upon the direct contact between the unlike propellants as well as the velocity difference to provide the primary atomization of the liquid propellant. With both element types, with liquid oxygen and gaseous propellants, the shearing action in the primary atomization can be affected by the reaction because the reaction rates of oxygen and hydrogen are relatively fast, and hence promote the existence of a flame even as far upstream as the exit of the oxidizer post. ${ }^{19}$ Flame in this location implies reaction temperatures and products exist there that separate the shearing streams differently than if the streams had not been reacting. However, examination of this separation in experiments and models to date suggest that near the element exit, the flame zone is not wide and the large scale motion driven by the large differential velocity is still responsible for the primary atomization of the oxidizer jet. ${ }^{16,17}$

For this reason, non-reacting ("cold") flows were selected in this university-led project for use as an initial investigation into scaling and throttling of the injection and atomization processes. Cold flow tests provide a much more benign environment to measure characteristics of the injection and atomization processes than can be measured in a hot-fire environment, while also maintaining the most reasonable semblance to the real environment. 


\section{Primary Atomization for LRE Coaxial Elements}

Initial studies of primary atomization of round jets suggest that the appropriate physics can be captured by comparing the jet Reynolds number $(R e)$ to a form of the jet Weber number (We), such as shown in Fig. 3. Identified on Fig. 3 are the generally accepted regions where flow at the oxidizer jet exit becomes turbulent assuming it is uniform and free of oscillations $(R e>2000-10,000)$, and where the atomization regime begins (We > 100). Note that the jet We defined in Fig. 3 is valid for a jet injected into a quiescent atmosphere, because the momentum includes only the jet velocity. Fig. 3 also includes estimates for some of the U.S.-built $\mathrm{O}_{2} / \mathrm{H}_{2}$ LREs that are currently of interest to the NASA Exploration Mission, including RL10, J-2, and Space Shuttle Main Engine (SSME) main injector and fuel-pump preburner. The two SSME injectors are shown over a normal operating range that extends from $109 \%$ power level to $65 \%$. Table I includes some information on these engines.

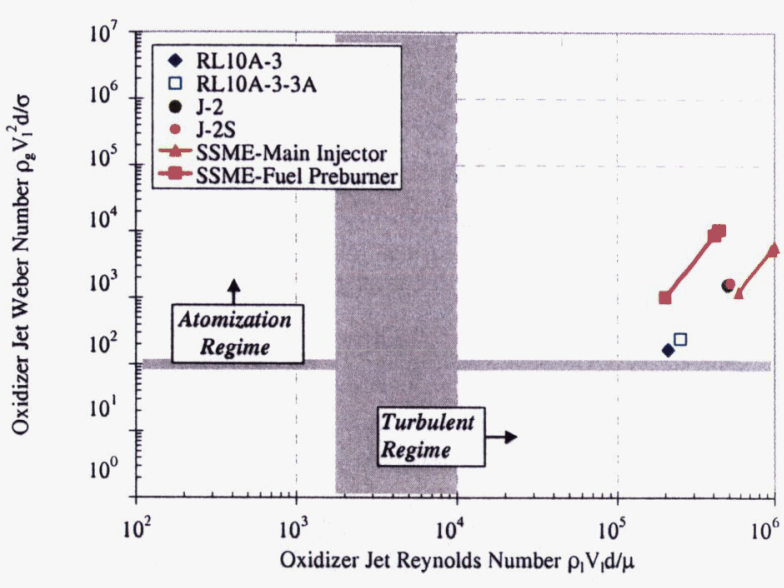

\begin{tabular}{|c|c|c|c|c|c|}
\hline & & RL10A-3 & J-2 & $\begin{array}{c}\text { SSME- } \\
\text { MI }\end{array}$ & $\begin{array}{c}\text { SSME- } \\
\text { FPB }\end{array}$ \\
\hline Vacuum Thrust & klbf & 15 & 230 & 512 & N/A \\
\hline Isp & sec & 427 & 430.7 & 452.3 & N/A \\
\hline $\begin{array}{c}\text { Chamber } \\
\text { Pressure (Face) }\end{array}$ & psia & 302 & 787 & 2988 & 5096 \\
\hline $\begin{array}{c}\text { Mixture Ratio } \\
\text { oxidizer mass } \\
\text { flowrate/element }\end{array}$ & Ibm/sec & 0.136 & 0.740 & 1.452 & 0.274 \\
\hline $\begin{array}{c}\text { oxidizer exit } \\
\text { orifice diameter }\end{array}$ & inches & 0.079 & 0.182 & 0.188 & 0.089 \\
\hline
\end{tabular}

Figure 3. Comparison of jet $R e$ and $W e$ and typical O2/H2 LRE coaxial injector elements. Table I. Representative LRE data

The meaning of "atomization regime" when this We $>100$ is the subject of some disagreement. Usually, the meaning is that the disintegration of the jet is complete within a short distance from the discharge orifice exit, ${ }^{20}$ or the jet disrupts completely at the exit. ${ }^{21}$ Thus, primary atomization of the jet occurs within a short distance from the exit. Others have suggested that the exterior of the jet does not have the same characteristics as the interior; i.e., the exterior begins to break and separate while the interior remains intact for some further distance. For the present work, this needs further clarification. ${ }^{22}$ Chigier suggests a "fiber-type" regime, in which fibers are peeled off the liquid surface and break into drops much smaller than the jet diameter. ${ }^{23}$

The injectors for the LREs shown in Fig. 3 are shown to operate well into the turbulent regime, but only one or two orders of magnitude into the atomization regime. However, the jet We shown in Fig. 3, and as typically used in jet atomization literature, may be misleading in regard to the injector elements as are typically used in these LREs. As discussed in Sec. I.B., the primary atomization of the liquid jet in these elements is largely dependent upon the velocity differential between the coaxial, annular gaseous fuel and the central liquid oxidizer, rather than just on the liquid jet velocity itself. This change in the definition of the aerodynamic forces on the liquid jet has a profound effect on the We, as shown in Fig. 4. The aerodynamic $W e \sim 10^{4}$ to $10^{6}$ for the injector elements from the rocket engines shown in Table I are now 2 to 3 orders of magnitude greater than on Fig. 3. Along with the jet $R e \sim$ $10^{5}$ to $10^{6}$, these numbers - as has been pointed out many times - are many orders of magnitude from typical primary jet disintegration studies. ${ }^{20}$ The LRE elements operate 1 to 3 orders of magnitude in Re beyond what is usually considered the onset of turbulent flow regime, and 2 to 4 orders of magnitude in We beyond what is usually considered the atomization regime. 


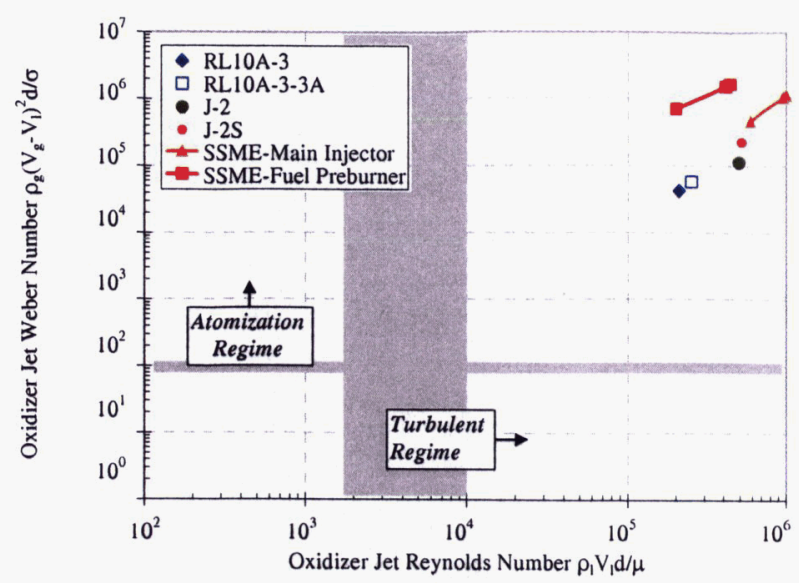

Figure 4. Comparison of jet $R e$ and aerodynamic jet We and typical O2/H2 LRE coaxial injector elements.

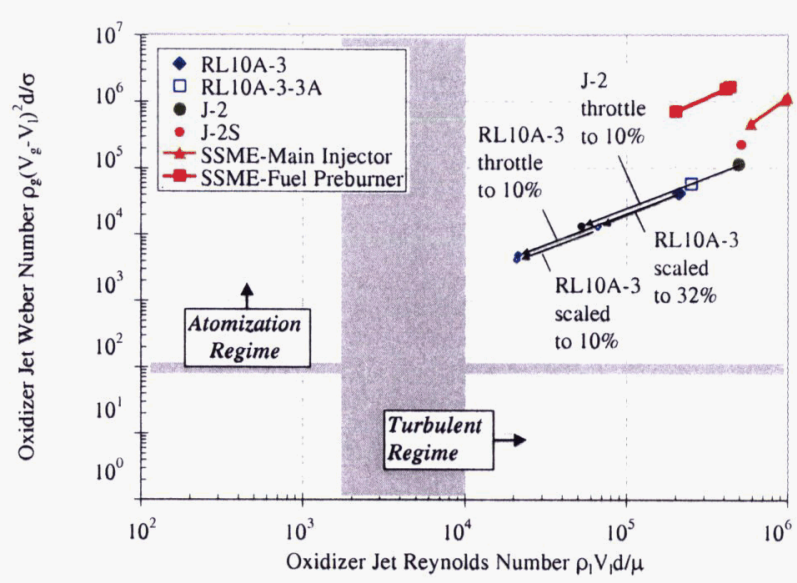

Figure 5. Fig. 4 including scaling and throttling of RL10 and J-2 injection elements.

Even when these injectors are scaled or throttled excessively, the conditions in the injector do not approach non-turbulent or non-atomization regimes, as illustrated in Fig. 5. In this figure, the RL10 and J-2 engines were artificially throttled according to conditions from normal $100 \%$ operating conditions by reducing the mass flow rates and differential pressures across the injectors according to the known states of the propellants in the injectors, which reduces the combustion chamber pressure in a chamber with a fixed throat area. These throttled conditions are likely to be different from actual throttling of the real engines because of limitations due to allowable operating regimes of the turbomachinery (e.g., mixture ratio in the combustion chamber is not likely to remain constant as it was in Fig. 5). The RL10 engine injector was scaled geometrically by reducing the injector element size, flow rates, and throat area proportionally, so that the chamber pressure remained constant. Thus, the RL10 scaled to $32 \%$ has the same flow rate as throttled to $10 \%$. Fig. 5 shows that, even throttled or scaled dramatically, these LRE elements operate $\sim 1$ order of magnitude in $R e$ beyond what is usually considered the onset of turbulent flow regime, and 1 to 2 orders of magnitude in We beyond what is usually considered the atomization regime. The figure also suggests that, at least for the process of primary atomization, scaling and throttling follow similar trends when considering jet turbulence and the ratio of momentum to surface tension forces represented by the aerodynamic We.

The primary atomization regimes of jets have been distinguished on other plots as well. Fig. 6 compares the jet Ohnesorge number $(\mathrm{Oh})$ and the jet $R e$. The lines between distinguishing regimes are described by Reitz and Bracco, ${ }^{21}$ based on the original work of Ohnesorge and later modified by Miesse. ${ }^{20,24}$ The locations of the regimes are different than those shown in the figures in [20], which are believed to be in error. Note that the Oh number in Fig $6, \mu /\left(\rho_{1} \sigma \mathrm{d}\right)^{0.5}$ is often represented as $W e^{0.5} / R e$, which is misleading because the $W e$ in this case would be defined as $\rho_{1} V_{1}^{2} d / \sigma$, rather than $\rho_{\mathrm{g}} \mathrm{V}_{1}^{2} \mathrm{~d} / \sigma$ as used in Fig. 3 or $\rho_{\mathrm{g}}\left(\mathrm{V}_{\mathrm{g}}{ }^{2}-\mathrm{V}_{1}{ }^{2}\right) \mathrm{d} / \sigma$ as used in Figures. 4 and 5. Fig. 6 shows that the LREs represented operate in the atomization regime on the border with the second wind-induced regime. Scaling and throttling the RL10 and J-2 engines on this plot as in Fig. 5 is shown in Fig. 7. For these cases, primary atomization of these throttled and scaled elements changes regimes. Throttling the J-2 to $10 \%$ changed the primary atomization classification from the atomization regime to the first wind-induced regime. Also, unlike on Fig. 5, scaling and throttling did not result in similar characteristics. The RL10 example shown on Fig. 7 suggests that scaling and throttling will cause diverging primary atomization characteristics. The reason for this is that the $O h$ includes the jet diameter $d$ along with propellant properties, but not any characteristic jet velocity. 


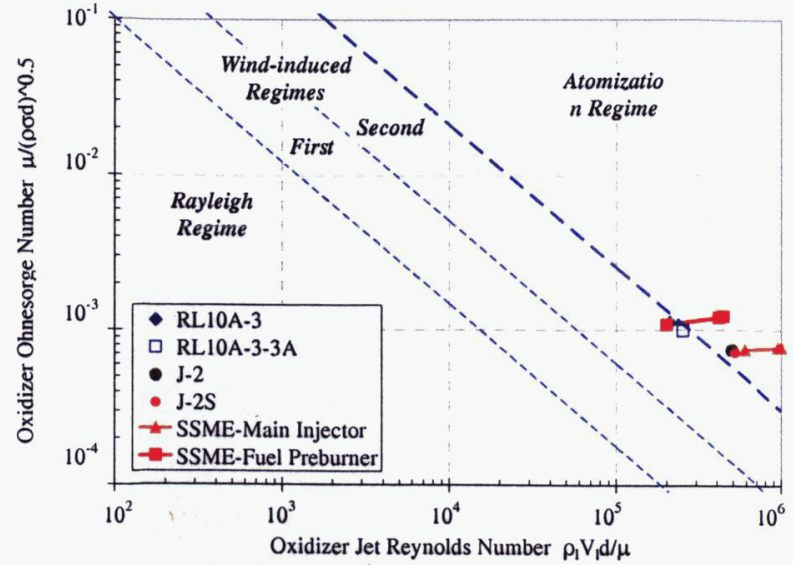

Figure 6. Primary atomization regimes identified on jet $R e$ versus $O h$.

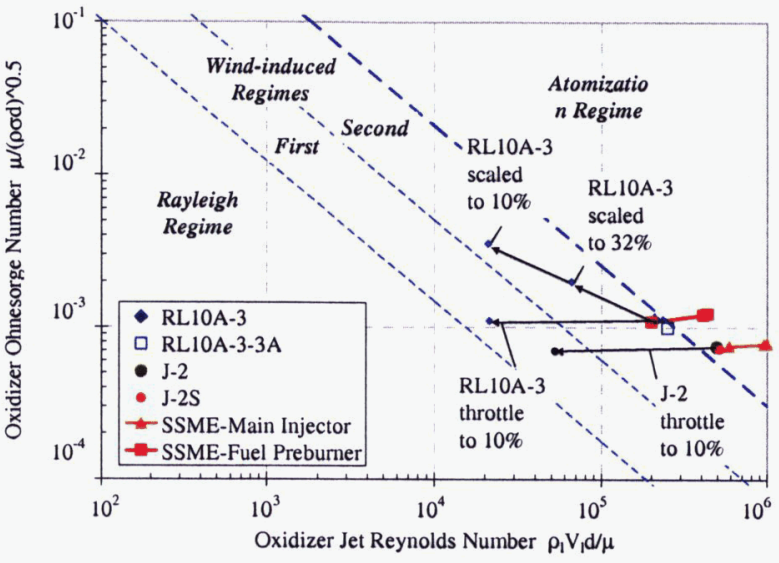

Figure 7. Fig. 6 including scaling and throttling of RL10 and J-2 injection elements.

Another plot distinguishing regimes of primary atomization, attributable to Chigier, ${ }^{23}$ is shown in Fig. 8 . This plot is the inverse of Fig. 4, but on this plot Chigier has identified 4 different regimes, although at much lower jet $R e$ and aerodynamic jet We than are typical for the rocket engines listed. Chigier has claimed that typical LRE injector elements such as used in the SSME operate in the fiber-type regime, ${ }^{23}$ which except for the fuel-pump preburner of the SSME is possible simply by extending the line dividing the fiber-type regime and the superpulsating regime. Throttling and scaling the RL10 and J-2 engines on this plot as in Fig. 5 is shown in Fig. 9. Unless there are other regimes present at these $R e$ and $W e$ that have not been identified, it appears in these cases that the primary atomization of these throttled and scaled elements do not change physical regimes.

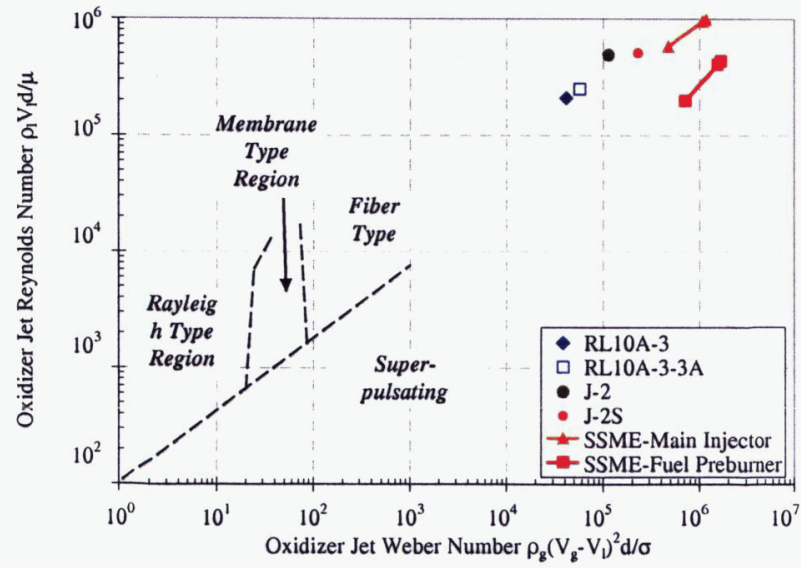

Figure 8. Primary atomization regimes identified on jet aerodynamic We versus $R e$.

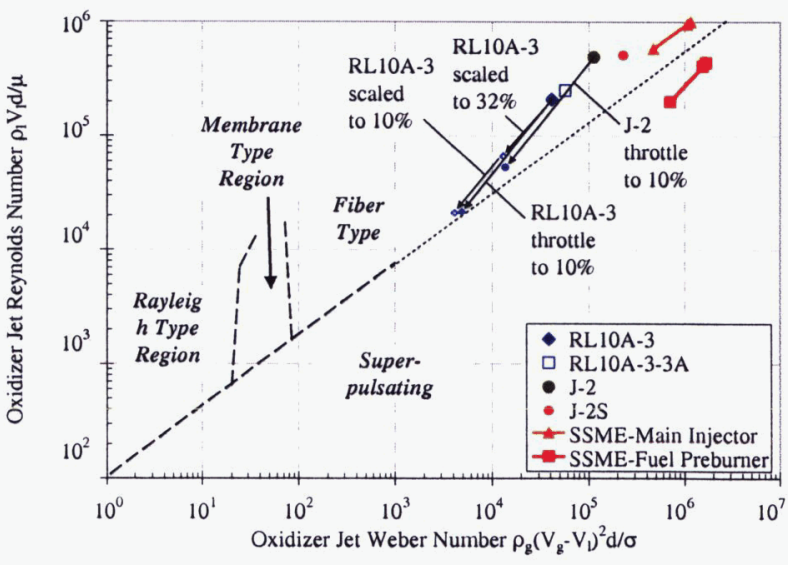

Figure 9. Fig. 8 including scaling and throttling of RL10 and J-2 injection elements.

Other researchers have suggested other groupings to fully capture all of the fluid mechanics of the injection and atomization processes. Farago claims that two more dimensional groupings are important for cold flow studies: density ratio and viscosity ratio of the propellants. ${ }^{25}$ Instead of the viscosity ratio, Vingert claims that the propellant injection velocity ratio is required. ${ }^{26}$ These relationships have not yet provided any distinguishing comparisons with the current data, but are still being evaluated.

A comparison of Figures 5, 7, and 9 shows that there is yet no definitive agreement of the primary atomization regimes for typical LRE coaxial injector elements, and especially as they are geometrically scaled or throttled. Typical injectors operated at their nominal design conditions are on the edge or well into atomization regimes for the primary jet breakup. However, in some cases, scaling or throttling these injectors may change the regime that describes the basic character of their primary atomization. It seems to these authors that scaling of LRE 
injectors can only be defensible if the basic physical processes, such as primary atomization, do not change regimes. Identifying the possibility of changing atomization regimes while throttling, on the other hand, is a valuable input to LRE development for NASA's lunar and exploratory missions.

\section{CUIP Task Description}

Cold flow testing has been used for injector characterization on numerous rocket injector development programs. ${ }^{1,2}$ These programs used cold flow tests to assess many characteristics such as injector hydraulic resistance, break-up lengths, droplet size and velocity distributions, spray angles and general spray morphology, and mass flux distributions. Injector research programs have also used cold flow simulations for injector configuration sensitivity studies and for numerical model validation and verification. Even with all of the past usage of cold flow characterization, there always remain questions of how to interpret cold flow injector measurements to predict hotfire injector performance. The immediate goals for this project are to study injector non-reacting fluid dynamics with changing geometric scale and operating conditions, and compare the scaled and throttled conditions to each other, not to hot fire conditions. Also, the initial characteristics to study include injection and primary atomization processes, which as discussed above are those least likely to be affected by the reaction. Later phases of this research program may move into hot-fire tests with the same injector scales, providing comparisons to the cold flow assessments.

Regimes predicting atomization and mixing are usually based on test data for low mass flow rates and (typically) for atmospheric test conditions. To better simulate the non-reacting flow conditions with a given LRE, lab-scale test conditions must be designed to provide high mass flow rates for both gas and liquid, and possibly even a controllable high-pressure environment. For gaseous fuel/liquid oxygen propellant combinations, non-reacting simulants used have typically been air and water, respectively. For a given gaseous fuel/liquid oxygen injector, a typical cold flow test involves running water through the liquid oxygen fluid circuit and high pressure air through the fuel fluid circuit. Traditionally, these tests are run with simulant flow rates matching that of the intended propellant flow rates, with both fluids issuing into the atmosphere. Because the safety concerns of working in a high-pressure, reacting environment have been removed, cold flow test articles allow for many diagnostic-friendly modifications. Longer run times of the simulants allow for a lower degree of uncertainty associated with injection measurements. Some programs have gone a step further and injected simulants into a pressurized chamber to match the effects chamber pressure has on atomization and mixing. ${ }^{27-29}$ A further step in hot-fire simulation is the use of liquid nitrogen in place of water, capturing the supercritical vaporization behavior indicative of using liquid oxygen as a propellant. ${ }^{16,17}$

\section{A. Chamber Design}

This task on the CUIP was initiated to study liquid rocket injector spray characteristics under various geometric scales and throttled conditions. The immediate goal was to select, design, fabricate, and characterize single injector elements in a high-pressure, cold flow environment. This environment will be attained using the Water/Nitrogen Injection Spray Test (WNIST) rig, currently in operation at the NASA MSFC East Test Area. Cross-country nitrogen feed lines allows WNIST to operate at chamber pressures up to $1200 \mathrm{psi}$. Fig. 10 shows the general layout of the WNIST rig. 

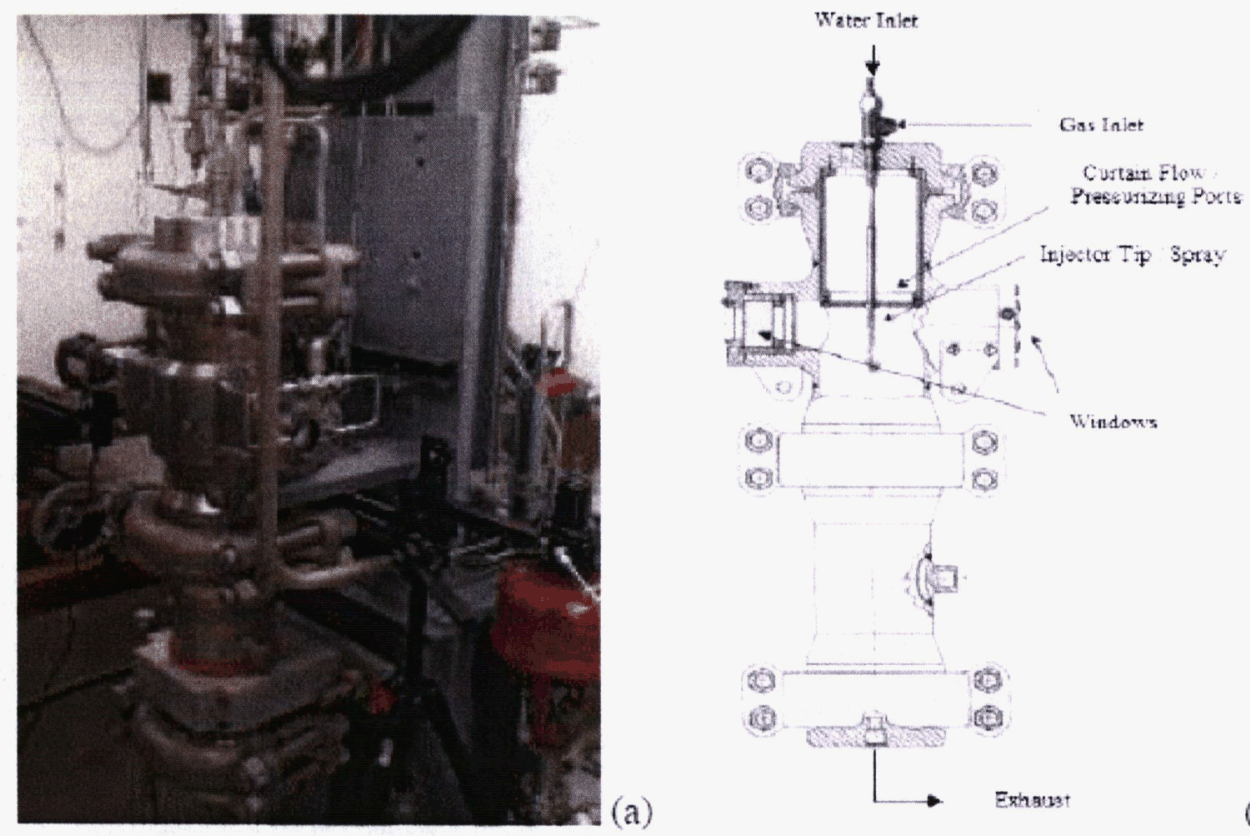

Figure 10. WNIST test rig and schematic.

Water $(\mathrm{H} 2 \mathrm{O})$ flow rates are delivered up to $1 \mathrm{lbm} / \mathrm{sec}$, and gaseous nitrogen $\left(\mathrm{GN}_{2}\right)$ flow rates are delivered up to $3 \mathrm{lbm} / \mathrm{sec}$. Various chamber sections provide optical access for shadowgraph, Phase Doppler Particle Analyzer (PDPA), and other diagnostics to the spray field. A real-time control system allows for readily throttling of both the mass flow rates and chamber pressures. A water heater modifies the $\mathrm{GN}_{2}$ temperature to control injected density for the gas simulant at a given chamber pressure.

\section{B. Injector Element Designs}

Injector elements planned for investigation in this task are coaxial types typical of injectors for LREs as listed in Table I, and of current interest in the LRE community. The first element type is a shear coaxial injector rated for $\sim 1 / 2$ to $1 \mathrm{lbm} / \mathrm{s}$ of LOX flowing through an inner post, and $\sim 0.15 \mathrm{lbm} / \mathrm{s}$ of $\mathrm{GH}_{2}$ annular flow. The other element type is a swirl coaxial injector, rated for the same LOX and GH2 flows as the shear coaxial element. Both elements are designed to work in a maximum pressure environment of $\sim 1200$ psia during nominal conditions. For each element type, three geometric scales were generated: a 'full-scale' element sized for full-flow conditions, a 'half-scale' element sized to deliver half of the flow rates, and a 'quarter-scale' element sized to deliver a quarter of the flow rates. The fluid properties used are those assuming liquid water for $\mathrm{LOX}$, and $\mathrm{GN}_{2}$ for $\mathrm{GH}_{2}$.

For this task, the injector cold flow characteristics will be studied for each of the scales given, over a 20:1 throttling range. The chamber pressure in the cold flow rig is varied to maintain reasonable gas velocities $(\sim 1000$ $\mathrm{ft} / \mathrm{sec})$ along with reasonable Mach numbers $(<0.5)$. This matching will be coupled with maintaining constant gas temperature, so that the density ratio of the predicted hydrogen flow and simulant nitrogen flow will be kept constant. While flow conditions such as mass flow rates and chamber pressures can be matched between propellants and simulants, fluid properties will be different, as shown in Table II. The densities and viscosities of liquid water and LOX are relatively close, but the surface tensions are much higher for water than for LOX. Consequently, the dimensionless groups, namely the Weber number, are generally smaller for water than for LOX for a given set of operating flow rates and pressures. 


\begin{tabular}{|c|c|c|}
\hline Liquid Properties & H2O & LOX \\
\hline Density $\left(\mathrm{lbm} / \mathrm{ft}^{3}\right)$ & $62.4\left(@ 68^{\circ} \mathrm{F}, 1 \mathrm{~atm}\right)$ & $49.9\left(@-243^{\circ} \mathrm{F}\right)-74.9\left(@-297^{\circ} \mathrm{F}\right)$ \\
\hline Viscosity $(\mathrm{lbm} / \mathrm{ft}-\mathrm{s})$ & $6.72^{*} 10^{-4}\left(@ 68^{\circ} \mathrm{F}, 1 \mathrm{~atm}\right)$ & $1.31^{*} 10^{-4}\left(@-297^{\circ} \mathrm{F}, 49 \mathrm{~atm}\right)$ \\
\hline Surface Tension $(\mathrm{lbf} / \mathrm{ft})$ & $4.99^{*} 10^{-3}\left(@ 68^{\circ} \mathrm{F}, 1 \mathrm{~atm}\right)$ & $8.22^{*} 10^{-4}\left(@-297^{\circ} \mathrm{F}, 49 \mathrm{~atm}\right)-$ \\
$(\mathrm{H} 2 \mathrm{O} / \mathrm{GN} 2$, and LOX/GH2$)$ & & $6.85^{*} 10^{-5}\left(@-225^{\circ} \mathrm{F}, 49 \mathrm{~atm}\right)$ \\
\hline Molecular Weight $(\mathrm{lbm} / \mathrm{lbmol})$ & $\mathbf{1 8 . 0 2}$ & $\mathbf{3 2}$ \\
\hline Gas Properties & GN2 & GH2 \\
\hline Molecular Weight $(\mathrm{lbm} / \mathrm{lbmol})$ & 28.02 & 2.02 \\
\hline
\end{tabular}

Table II. Comparison of $\mathrm{O} 2 / \mathrm{H} 2$ and water/N2 fluid properties. ${ }^{5}$

Predicted throttling ranges for each element and scale are shown in Figures. 11 to 14 in terms of the dimensionless groupings discussed in the previous section. Figures 11 and 12 show that the most of the cold flow ranges for all CUIP element scales fall inside the desired range of LRE operation, i.e., turbulent flow at the jet exits by $R e$ and the atomization regime by We. During throttling, all the injectors leave these regimes. Consequently, measurements of primary atomization for the CUIP elements are expected to be representative to typical LRE injector elements, but that relationship will not carry over the whole throttled range.

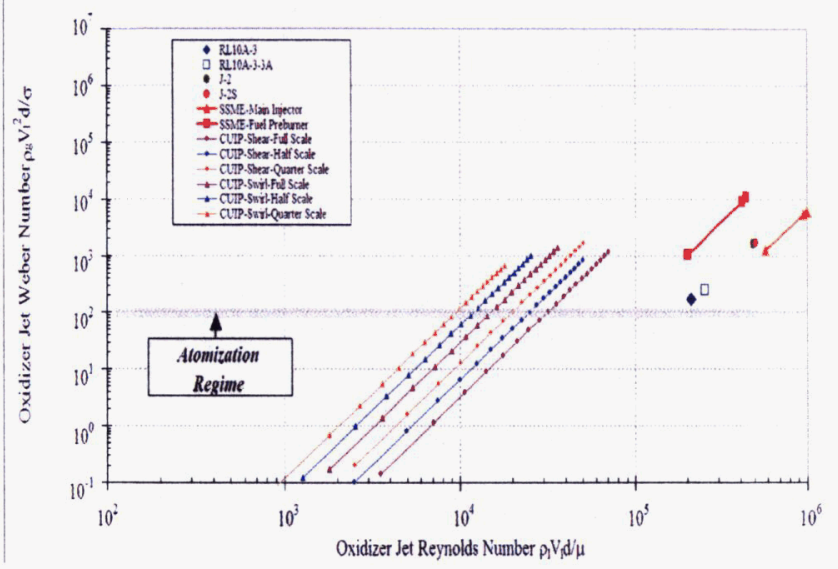

Figure 11. CUIP elements on jet $R e$ versus jet We.

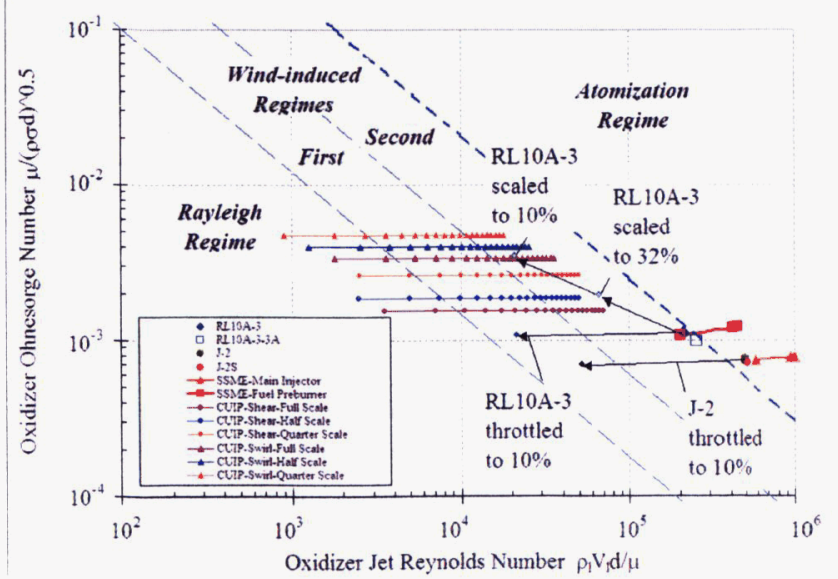

Figure 13. CUIP elements on jet $R e$ versus jet $O h$.

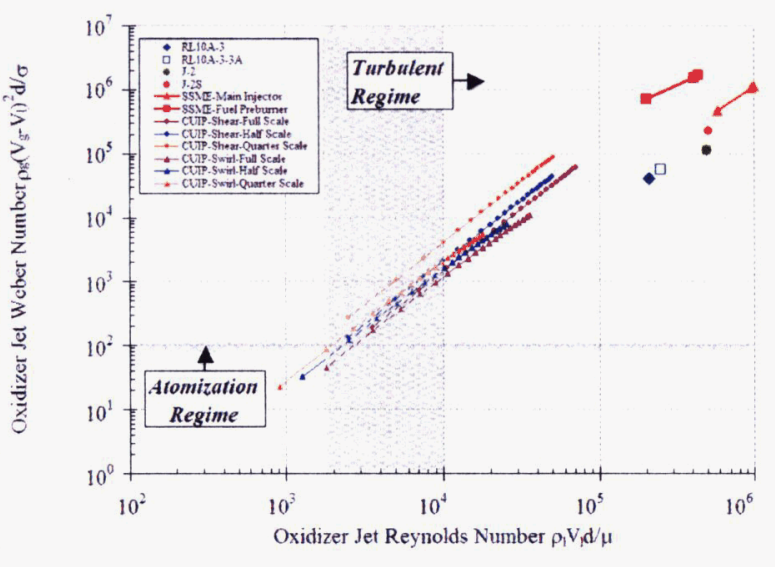

Figure 12. CUIP elements on on jet $R e$ versus aerodynamic jet We.

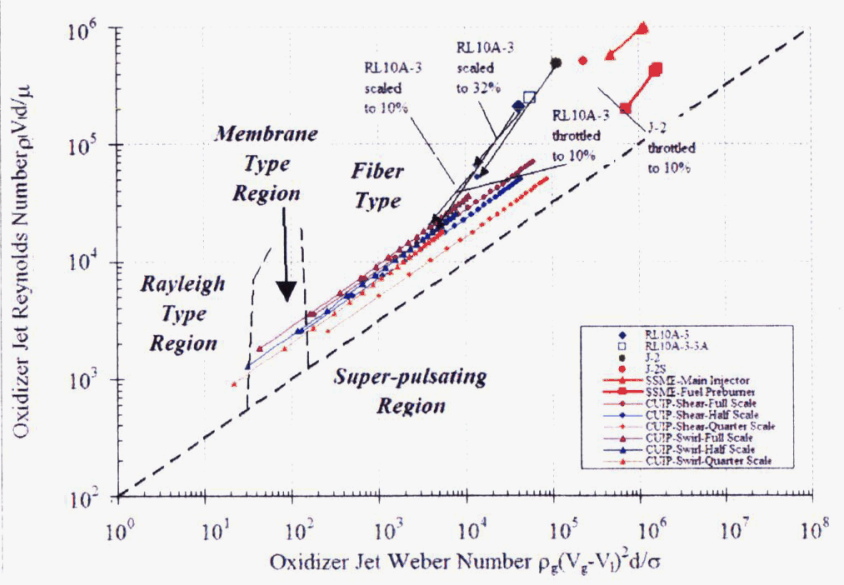

Figure 14. CUIP elements on on aerodynamic jet We versus jet $R e$.

Figures 13 and 14 show how the CUIP elements, using water/nitrogen simulants, compare to the atomization classifications shown previously in Figures. 7 and 9. Fig. 13 shows that the CUIP elements operate in the second wind-induced regime where some LREs operate, but are not able to achieve the atomization regime 
where other LREs operate. At best, the primary atomization data obtained by the CUIP injectors can be extrapolated to predict any LRE element behavior that coincides within the first-wind and second-wind induced regimes.

Fig. 14 shows that most of the CUIP elements operate within the same 'fiber-type' regime as the other LRE elements. This area, identified by Chigier to best categorize LRE injector operation, has large aerodynamic forces from shearing gas and low surface tension effects from the liquid oxidizer. While not matching the $R e$ and We of the LRE elements exactly, the CUIP injector elements can operate within the same atomization regime as the LRE elements. This indicates that the CUIP injectors can correctly simulate the fiber-type atomization process and characterize scaling and throttling of real LRE injectors.

\section{Preliminary Test Plans}

Initial phases of this cold flow work will evaluate how the CUIP element spray features vary with nondimensional groupings as presented in previous figures. The review on primary atomization of typical LRE coaxial elements to date has suggested current models do not adequately characterize primary atomization behavior at all representative LRE operating conditions. Thus, while data collected during this task will be compared against current atomization models, it is anticipated that new correlations will be generated. Once these relations are found, the effects of geometric scaling and throttling can be deduced from the expressions and explicitly stated. Diagnostics of these spray features will include shadowgraphs to measure spray angles and break-up lengths. The swirl-coaxial elements' will have acrylic sections for the LOX post; allowing for optical access to measure film thicknesses during atmospheric tests. Upon completion of atmospheric tests, the acrylic sections will be replaced with stainless steel and tested within a high-pressure chamber to quantify other spray features that are strongly dependant on the shearing gas density.

Other measurement techniques, including mechanical patternation to measure liquid and gas mass and mixture distributions and Phase Doppler Particle Analyzer (PDPA) to measure secondary atomization, are currently being developed by other parties to be implemented in the future. As with the primary atomization discussed in this paper, previous work with mass flux distributions and droplet behavior will be reviewed to determine whether the previous correlations can predict the desired regimes of LRE operation.

\section{CONCLUSIONS}

To date, there has not been a focused, systematic study on how LRE spray and combustion flows scale with combustor geometrical features. A successful research endeavor in this area could have significant influence on how LREs are developed in the future. One method, geometric scaling, is a simple procedure to generate subscale hardware for development.

An encompassing, LRE combustor-based research program that evaluates the rationale and effectiveness of scaling is probably not affordable for the foreseeable future. Such a program would also certainly leave many unanswered questions. A more focused, mechanism-oriented evaluation of scaling of LRE combustion devices will be more beneficial to the LRE research and development community, and piecemeal, is more affordable. A significant evaluation of scaling by this method would require evaluation of all the individual processes in spray combustion, including injection, atomization, vaporization, mixing, reaction, and acceleration, as well as their interactions. Some of these processes will be easier to investigate than others. The use of partial modeling of complex systems is proposed. The research plan presented in this paper is focused on evaluating the scaling of injection and primary atomization processes, which appear most tractable to experimental methods readily available to university-level laboratories at the University of Alabama, Huntsville. While scaling is being evaluated, it is a simple matter to extend the study to include throttling of LRE combustors, which include the same processes and many of the same questions.

Scaling and throttling of injection and primary atomization processes was reviewed. Different means to classify the atomization regimes were shown, delineated by dimensionless parameters such as the Reynolds number, Weber number, and Ohnesorge number. Typical $\mathrm{O}_{2} / \mathrm{H}_{2} \mathrm{LRE}$ injectors with coaxial elements were included on many of the plots to put the LRE designs in perspective. No decisive agreement was found that could clearly classify the regimes of primary atomization these injectors occupied, especially as they were arbitrarily scaled or throttled. 
Shear coaxial and swirl coaxial LRE injector elements were proposed to be included in a cold flow examination of injection and primary atomization processes. The cold flow tests use non-volatile and less hazardous fluids as simulants instead of the real and reactive propellants. This aspect of partial modeling was deemed acceptable because the injection and primary atomization processes are those generally least affected by the reaction. Use of cold flow testing for examination of other spray combustion mechanisms may be less acceptable.

A high-pressure, controllable cold flow facility will be used with water and $\mathrm{GN}_{2}$ to simulate $\mathrm{LOX}$ and $\mathrm{GH}_{2}$, respectively. The fluid properties of water and $\mathrm{GN}_{2}$ will limit how well some of the conditions will match those of $\mathrm{LOX} / \mathrm{GH}_{2}$. However, if the injector elements can operate within the same atomization regimes with water/GN $\mathrm{GN}_{2}$ as those with $\mathrm{LOX} / \mathrm{GH}_{2}$, then the injection and primary atomization processes measured with simulants and the effects of scaling and throttling can be related to the actual propellant spray features.

\section{Acknowledgments}

This research program was funded by the Constellation University Institute Program (CUIP) under the direction of Claudia Meyer and Dr. Jeffrey Rybak.

\section{References}

${ }^{1}$ Dexter, C.E., Fisher, M.F., Hulka, J.R., Denisov, K.P., Shibanov, A.A., and Agarkov, A.F., "Scaling Techniques for Design, Development and Test," Liquid Rocket Thrust Chambers: Aspects of Modeling, Analysis, and Design, edited by V. Yang, M. Habiballah, J. Hulka, and M. Popp, Progress in Astronautics and Aeronautics, AIAA, Washington, DC, 2004, pp. 553-600.

${ }^{2}$ Fisher, S.C., Dodd. F.E., and Jensen, R.J., "Scaling Techniques for Liquid Rocket Combustion Stability Testing," Liquid Rocket Engine Combustion Instability, edited by V. Yang, and W. Anderson, Progress in Astronautics and Aeronautics, AIAA, Washington, DC, 1995, pp. 545-564.

${ }^{3}$ Penner, S.S., Chemical Problems in Jet Propulsion, Pergamon, London, 1957, pp 345-347, 376-388.

${ }^{4}$ Adams, R. H., "Wind Tunnel Testing Techniques for Gas-Particle Flows in Rocket Exhaust Plumes," AIAA Aerodynamic Testing Conference, AIAA Paper No. 1966-767, September, 1966.

${ }^{5}$ White, F.M., Fluid Mechanics, $4^{\text {th }}$ ed., McGraw-Hill, New York, 1999, Chap. 5.

${ }^{6}$ Penner, S.S., "Similarity Analysis for Chemical Reactors and the Scaling of Liquid Fuel Rocket Engines," Combustion Research and Reviews, AGARD Combustion Colloquium, Butterworths, London, 1955, pp. 140-162.

${ }^{7}$ Lindsey, J.T., Elam, S., Koblish, T., Lee, P., and McAufflie, D., "Internal Flow Measurements of the SSME Fuel Preburner Injector Element Using Real Time Neutron Radiography," 26 Joint Liquid Propulsion Conference, AIAA Paper No. 1990-2293, July, 1998.

${ }^{8}$ Penner, S. S., "On the Development of Rational Scaling Procedures for Liquid-Fuel Rocket Engines," Jet Propulsion, Feb. 1957, pp. 156-168.

${ }^{9}$ Sutton, G.P., and Biblarz, O., Rocket Propulsion Elements, $7^{\text {th }}$ ed., John Wiley \& Sons, Inc., New York, 2001, Chaps. 6 - 9.

${ }^{10}$ Spaulding, D.B., "The Art of Partial Modeling," $9^{\text {th }}$ International Symposium on Combustion, The Combustion Institute, 1963, pp. 833-843

"Lawhead, R.B., and Combs, L.P., "Modeling Techniques for Liquid Propellant Rocket Combustion Processes," $9^{\text {th }}$ International Symposium on Combustion, 1963, pp. 973-981.

${ }^{12}$ Calhoon, D.F., Kors, D.L., and Gordon, L.H., “An Injector Design Model for Predicting Rocket Engine Performance and Heat Transfer," AIAA/SAE $9^{\text {th }}$ Propulsion Conference, AIAA Paper No. 1973-1242, November, 1973. p66.

${ }^{13}$ Harrje, D.T., and Reardon, F.H., "Liquid Propellant Rocket Combustion Instability," NASA SP-194, 1972, Figure 2.3.3a, 
${ }^{14}$ Ito, J.I., "Propellant Injection Systems and Processes," Liquid Rocket Thrust Chambers: Aspects of Modeling, Analysis, and Design, edited by V. Yang, M. Habiballah, J. Hulka, and M. Popp, Progress in Astronautics and Aeronautics, AIAA, Washington, DC, 2004, pp. 1-18.

${ }^{15}$ Hulka, J. and Makel, D., "Liquid Oxygen/Hydrogen Testing of a Single Swirl Coaxial Injector Element in a Windowed Combustion Chamber," AIAA Paper No. 93-1954, June 1993.

${ }^{16}$ Mayer, W., and Krülle, G., "Rocket Engine Coaxial Injector Liquid/Gas Interface Flow Phenomena," Journal of Propulsion and Power, Vol. 11, No. 3, May-Jun. 1995, pp. 513-518.

${ }^{17}$ Chehroudi, B., Talley, D., Mayer, W., Branam, R., Smith, J., Schik, A., and Oschwald, M. "Understanding Injection into High Pressure Supercritical Environments" 5th International Conference on Liquid Rocket Propellant, Chattanooga, 2003.

${ }^{18}$ Lawver, B.R., "High-Performance $\mathrm{N}_{2} \mathrm{O}_{4}$ /Amine Elements - Blowapart, Final Report," Report 14186-DRL-5, Contract NAS9-114186, Sacramento, CA, March 1979.

${ }^{19}$ Mayer, W, Schik, A., Vielle, B., Chauveau, C., Gökalp, I., Talley, D., and Woodward, R., "Atomization and Breakup of Cryogenic Propellants Under High-Pressure Subcritical and Supercritical Conditions," Journal of Propulsion and Power, Vol. 14, No. 5, Sept.-Oct. 1998, pp. 835-842.

${ }^{20}$ Lefebvre, A.H., Atomization and Sprays, Hemisphere Publishing Company, c. 1989, pp 37-59.

${ }^{21}$ Reitz, R.D., and Bracco, F.V., "Mechanisms of Breakup of Round Liquid Jets," Chapter 10, Encyclopedia of Fluid Mechanics, edited by N.P. Cheremisinoff, Vol. 3, Gulf, 1986, pp.233-249.

${ }^{22}$ interior vs exterior jet atomization

${ }^{23}$ Chigier, N.A., "The Physics of Atomization," Plenary Lecture, Proceedings of the Fifth ICLASS, Gaithersburg, MD, 1991.

${ }^{24}$ Miesse, C.C., "Correlation of Experimental Data on the Disintegration of Liquid Jets," Ind. Eng. Chem, Vol. 47, No. 9, 1955, pp.1690-1701.

${ }^{25}$ Farago, Z., and Chigier, N., "Morphological Classification of Disintegration of Round Liquid Jets in a Coaxial Air Stream," Atomization and Sprays, Vol. 2, 1992, pp. 137-153.

${ }^{26}$ Vingert, L., Gicquel, P., and Lourme, D., "Coaxial Injector Atomization," Liquid Rocket Engine Combustion Instability, edited by V. Yang, and W. Anderson, Progress in Astronautics and Aeronautics, AIAA, Washington, DC, 1995, pp. 145-189.

${ }^{27}$ Cohn, R.K., Strakey, P.A., Bates, R.W., Talley, D.G., Muss, J.A., and Johnson, C.W., "Swir] Coaxial Injector Development," $41^{s t}$ Aerospace Sciences Meeting and Exhibit, AlAA Paper No. 2003-0125, January, 2003.

${ }^{28}$ Strakey, P.A., Cohn, R.K., and Talley, D.G., "The Development of a Methodology to Scale between Cold-flow and Hot-fire Evaluation of Gas-centered Swirl Coaxial Injectors," 52 nd JANNAF Propulsion Meeting, 10-14 May 2004.

${ }^{29}$ Kim, D., Jeong, W., Im, J.H., and Yoon, Y., "The Characteristics of Swirl Coaxial Injector Under Varying Geometric and Environmental Conditions," 40 th Joint Liquid Propulsion Conference, AIAA Paper No. 2004-3521, July, 2004. 